\title{
Internacionalización de la mediana empresa familiar Ecuatoriana: el rol de la orientación a la administración y flexibilidad estratégica del equipo gerencial
}

\section{Internationalization of Ecuadorian family businesses: the role of management guidance and strategic flexibility of the management team}

\author{
Esteban Andrade Rodas \\ Universidad Internacional del Ecuador, Ecuador
}

Autor para correspondencia: fandrade@uide.edu.ec

Fecha de recepción: 07 de Agosto de 2017 - Fecha de aceptación: 10 de Agosto de 2017

Resumen: A pesar de la importancia de la empresa familiar y su rol dominante en la economía mundial, el conocimiento empírico sobre su internacionalización resulta escaso, evidenciándose en la literatura la necesidad de estudiarlo. Entre los factores que motivan la internacionalización se ha identificado su orientación a la administración y la flexibilidad estratégica de su equipo gerencial. A partir de una muestra tomada en medianas empresas familiares ecuatorianas, este trabajo investiga la influencia de la orientación de la administración y la flexibilidad estratégica del equipo gerencial en la actividad exportadora, misma que es evaluada por el grado de internacionalización. Los hallazgos revelan la existencia de una relación positiva de estas variables en el grado de internacionalización y además como la orientación a la administración acoplada con la flexibilidad estratégica del equipo gerencial influye de manera positiva en la actividad exportadora. Los resultados evidencian la importancia de estos factores en la internacionalización de la mediana empresa familiar ecuatoriana, ofreciendo elementos valiosos para su administración y supervivencia.

Palabras Claves: empresa familiar, internacionalización; orientación a la administración; equipo gerencial; actividad exportadora; pequeña y mediana empresa ecuatoriana

\begin{abstract}
Despite the importance of family business and its dominant role in the world economy, the empirical knowledge about its internationalization is scarce, becoming evident in the literature with its necessity of being studied. Among the factors that motivate the internationalization, its orientation to the management and the strategic flexibility of its management team have been identified. Based on a sample taken in Ecuadorian family-owned medium-sized enterprises, this paper investigates the influence of the administrative orientation and the strategic flexibility of the management team in the export activity, which is evaluated by the level of internationalization. The outcomes reveal the presence of a positive relationship between these variables in the level of internationalization and, in addition, the orientation to the administration coupled with the strategic flexibility of the management team influence in a positive way the export activity. The results evince the importance of these factors in the internationalization of the Ecuadorian family-owned medium-sized enterprises, offering valuable elements for its management and survival.
\end{abstract}

Key words: family firm; internationalization; stewardship orientation; top management team; export activity; medium sized ecuadorian enterprise 


\section{Introducción}

La empresa familiar constituye la forma más antigua y extendida de negocio en el mundo, ofreciendo un gran aporte al empleo y economía de las naciones (Alkaabi \& Dixon, 2014; Ocampo Figueroa, Alarcón Osuna, \& Fong Reynoso, 2014). La variable "familia" no ha sido frecuentemente considerada en las investigaciones relacionadas al campo empresarial, e incluso algunos investigadores creen que omitirla puede llevar a conclusiones incompletas y muchas veces engañosas (Mitter, Duller, Feldbauer-Durstmüller, \& Kraus, 2014; Sciascia, Mazzola, Astrachan, \& Pieper, 2012). A diferencia de la empresa no familia, la empresa familiar es una organización que combina los ámbitos de la empresa y la familia impactando de manera directa en la misma y determinando una importante diferencia con empresas que cuentan con otro tipo de propiedad (Craig, Dibrell, \& Garrett, 2014).

Estudios académicos han centrado sus esfuerzos en investigar las metas económicas y el desempeño de las empresas; sin embargo, estos criterios tienen una limitada utilidad para la familia propietaria y sus administradores, quienes se esfuerzan por lograr un balance entre las metas económica y no económicas cuando diseñan estrategias, administran y toman decisiones operativas (Chrisman, Chua, \& Sharma, 2005). Dada esta dualidad, la empresa familiar requiere de estructuras de gobierno que unan la complejidad de los sistemas que conforman la familia y la empresa lo que ocasiona que el gobierno de la empresa se constituya en un desafío particular y difiera de la empresa no familiar (Calabrò \& Mussolino, 2013). La empresa familiar desarrolla una estructura de gobierno que promueve la cohesión y una visión compartida dentro de la familia, buscando reducir potenciales y perjudiciales conflictos (Calabrò \& Mussolino, 2013). Muchas de las empresas más grandes y exitosas del mundo tienen carácter familiar, sin embargo, éstas no pueden ser comparadas con la mediana empresa familiar porque su tamaño tiene importantes efectos en la estructura de la organización (Swoboda \& Olejnik, 2013).

\section{Internacionalización de la Mediana Empresa Familiar}

El crecimiento de la economía mundial y la globalización han estimulado a las empresas de todos los tamaños y tipos de propiedad a expandir sus operaciones a nivel internacional, convirtiéndose en una respuesta estratégica a la competencia internacional, independientemente de su tamaño, sector industrial o país de origen (Calabrò \& Mussolino, 2013; Cerrato \& Piva, 2012). Desde que las empresas ya no son protegidas de competidores externos, ésta se ha incrementado tanto a nivel de mercado doméstico como internacional, ocasionando que la medianas empresas evalúen incursionar en mercados externos (Calabrò \& Mussolino, 2013). De acuerdo a Le y Valadkhani (2014) las empresas que exportan son más productivas y pueden llegar a ser más competitivas, reportando mayores niveles de eficiencia que las no exportadoras.

La internacionalización resulta especialmente crítica para la mediana empresa familiar dado que se trata de un proceso difícil y exigente que requiere de recursos humanos financieros y logísticos, así como de una clara estrategia para avanzar (Calabrò \& Mussolino, 2013). Sus limitados objetivos de crecimiento, su cultura adversa al riesgo y el deseo de evitarlo, junto con la dotación limitada de recursos financieros además restringidos por el poco deseo de compartir la propiedad con miembros no familiares, limitadas capacidades gerencias de administradores con poca o ninguna experiencia internacional, la insuficiente disposición a aceptar talentos 
externos ajenos a la familia propietaria y la falta de vínculos con redes externas, sumado a problemas culturales y políticos, hacen que se muestre menos proclive a internacionalizarse y siga un camino lento, cauteloso y poco estructurado, sintiendo temor a perder el control sobre el negocio o la riqueza de la familia (Alkaabi \& Dixon, 2014; Calabrò \& Mussolino, 2013; Cerrato \& Piva, 2012; Kontinen \& Ojala, 2011; Swoboda \& Olejnik, 2013).

Para la empresa familiar es motivo de preocupación el lograr equilibrar los intereses de la familia y la empresa, y muchas veces las metas no económicas pueden ser más importantes (Cerrato \& Piva, 2012; Chrisman et al., 2005). De ahí que los procesos y estrategia de internacionalización difieran entre la empresa familiar y las que no lo son, y en general con aquellas que tienen una estructura de propiedad diferente (Cerrato \& Piva, 2012; Kontinen \& Ojala, 2011; Piva, Rossi-Lamastra, \& De Massis, 2013; Swoboda \& Olejnik, 2013).

Al parecer, un cierto grado de orientación internacional, orientación al riesgo y orientación hacia las personas es necesario para el éxito en los mercados extranjeros (Swoboda \& Olejnik, 2013). La empresa debe creer en el potencial de los negocios internacionales y estar dispuesta a perseguir inciertas oportunidades en mercados externos (Swoboda \& Olejnik, 2013). Es necesario que la pequeña y mediana empresa logre mayor consciencia de la importancia de la internacionalización como una potencial forma de crecimiento con la cual mejore su rentabilidad y posibilidades de supervivencia (Cerrato \& Piva, 2012). Por esto, la decisión de internacionalizarse por parte de una empresa, es algo que la mediana empresa con carácter familiar, podría tener necesidad de enfrentar en su esfuerzo por crear valor para la organización, dejando de lado aspectos tradicionales como su tamaño o edad (Calabrò \& Mussolino, 2013).

Si bien la internacionalización es un tópico estudiado dentro de la gestión empresarial y con una creciente atención, la internacionalización de la empresa familiar constituye una importante área de estudio con escasas investigaciones académicas, en las cuales el involucramiento de la familia ha sido prácticamente olvidado, sin considerar cómo el carácter familiar influye en la internacionalización así como los factores que la motivan (Acedo, Casillas, \& Moreno, 2008; Calabrò \& Mussolino, 2013; Cerrato \& Piva, 2012; Fernández \& Nieto, 2005; Graves \& Thomas, 2008; Hiebl, 2013; Kontinen \& Ojala, 2010, 2012; Pukall \& Calabrò, 2014; Sciascia et al., 2012; Segaro, Larimo, \& Jones, 2014; Yeoh, 2014). Estudios realizados no han considerado el papel de la familia en la iniciativa empresarial internacional [international entrepreneurship] y las dificultades que ésta enfrenta, porque la mayoría no diferencia entre empresas familiares y no familiares (Abdellatif, Amann, \& Jaussaud 2010; Sciascia et al., 2012).

\section{Propósito}

De acuerdo a la literatura surge la necesidad de investigar los factores que influyen en la internacionalización de la pequeña y mediana empresa familiar, identificándose como los principales a la propiedad, gobierno y equipo gerencial (Acedo et al., 2008; Cerrato \& Piva, 2012; Segaro, 2012; Segaro et al., 2014; Zahra, Neubaum, \& Naldi, 2007). El presente estudio busca examinar empíricamente la relación entre la orientación a la administración [stewardship orientationk] que representa al gobierno de la empresa, y la flexibilidad estratégica del equipo gerencial que constituye una variable que forma parte del equipo gerencial, como factores que inciden en la internacionalización y su efecto en la iniciativa empresarial internacional, 
representada por el grado de internacionalización, en la mediana empresa familiar ecuatoriana. Para cumplir este propósito, el presente estudio es cuantitativo, bajo el paradigma positivista, siguiendo un enfoque de investigación deductivo y realizado en un solo momento del tiempo por lo que tiene carácter de transversal. En Ecuador no se han identificado investigaciones de este tipo, por lo que se busca llenar el vacío existente en la literatura de la empresa familiar ecuatoriana, contribuyendo indirectamente a su éxito y del país (Salomón, 2010).

\section{Gobierno de la Empresa Familiar}

El gobierno de la empresa es un factor por el cual la participación de la familia propietaria puede ser evaluada, más aún cuando un alto involucramiento de la familia puede representar temor a la destrucción de la riqueza y es vista como una fuente de resistencia al cambio y deseo de mantener el estado actual de las cosas (Cerrato \& Piva, 2012; Garcia-Castro \& Aguilera, 2014). El éxito de la empresa familiar está fuertemente influenciado por la manera en que la familia se ha organizado y como gestiona su relación con la empresa (Suess, 2014).

El gobierno de la empresa ha sido predominantemente estudiado usado la teoría de la agencia [agency theory], sin embargo, su validez ha sido cuestionada en el contexto de la empresa familiar, considerando a la teoría de la administración [stewardship theory] propuesta por Davis, Schoorman, y Donaldson (1997) y dentro de la misma la orientación a la administración [stewardship orientation], como una visión alterna que permite explicar los efectos positivos de la propiedad de la familia y conocer como los administradores defienden los intereses de los propietarios, buscando mecanismos para lograr bienestar de largo plazo (Kontinen \& Ojala, 2012; Miralles-Marcelo, Miralles-Quirós, \& Lisboa, 2014; Mitter et al., 2014; Sciascia et al., 2012; Segaro et al., 2014).

\section{Teoría de la Agencia en el Contexto del Gobierno de la Empresa Familiar}

La Teoría de la Agencia describe las relaciones entre dos grupos: el principal y el agente, abordando la relación desde una perspectiva conductual y de gobierno (Madison, Holt, Kellermanns, \& Ranft, 2016). De acuerdo a la economía, la teoría de la agencia sugiere que los agentes elegirán el comportamiento egoísta y oportunista cuidando sus propios intereses en lugar de un comportamiento dirigido a maximizar los intereses del principal (Davis et al., 1997; Madison et al., 2016). Por tanto, el principal implementará mecanismos de gobierno para monitorear y frenar el comportamiento oportunista del agente, con el propósito de frustrar cualquier comportamiento del agente no alineado con el interés del principal y por esta vía aumentar el rendimiento de la empresa (Madison et al., 2016).

La propiedad de la familia ha sido descrita como un mecanismo eficaz de gobernanza organizacional, debido a que reduce los problemas asociados con la separación de la propiedad y la gestión, sin embargo, hay estudios que sugieren lo contrario, por cuanto la dinámica familiar y los conflictos que de ella surgen, son difíciles de controlar y se ha demostrado de manera empírica que está asociado a una menor productividad (Calabrò \& Mussolino, 2013; Madison et al., 2016). Por ello, la propiedad de la familia puede facilitar los problemas de agencia como la incapacidad de tomar sólidas decisiones empresariales debido a un excesivo apego emocional o la falta de un sucesor familiar cualificado (Madison et al., 2016). 


\section{Teoría de la Administración en el Contexto del Gobierno de la Empresa Familiar}

La teoría de la administración, al igual que la teoría de la agencia, aborda la relación desde una perspectiva de comportamiento y de gobierno (Madison et al., 2016). Debido a sus orígenes en la sociología y sicología, la teoría de la administración representa un modelo más humanista del hombre, frente a la visión económica de la teoría de agencia (Madison et al., 2016). La teoría de la administración se enfoca en el compromiso y dedicación de los gerentes para con la organización ofreciendo una visión alternativa, la cual se sustenta en la idea que los gerentes en su rol de administradores tienen un alto sentido del deber hacia la organización y buscan el bienestar colectivo antes que el bienestar individual con el objetivo de mejorar el desempeño de la organización mirando la utilidad de largo plazo y no un comportamiento oportunista de corto plazo, y por tanto, su accionar se alineará de manera natural con los intereses del principal (Alkaabi \& Dixon, 2014; Davis et al., 1997; Kontinen \& Ojala, 2012; Madison et al., 2016; Miralles-Marcelo et al., 2014; Sciascia et al., 2012; Segaro et al., 2014). La teoría de la administración ha demostrado que una relación y procesos familiares efectivos contribuyen al desempeño de la empresa, aportando positivamente a su internacionalización (Eddleston, Kellermanns, \& Zellweger, 2012).

A diferencia de los mecanismos tradicionales orientados al control que están presentes en la teoría de la agencia, el gobierno de la empresa familiar bajo la teoría de la administración fomenta la cooperación, empodera y motiva a los empleados, ocasionando un comportamiento a favor de la organización y mejorando el desempeño de la empresa (Davis et al., 1997; Madison et al., 2016). El gobierno por medio de la teoría de la administración se ha vinculado con niveles más altos de emprendimiento en las empresas familiares, pues éstas son capaces de identificar y explotar oportunidades empresariales cuando las ideas críticas de los miembros de la familia se combinan con perspectivas de miembros no familiares y de manera conjunta son capaces de participar en el proceso de toma de decisiones (Madison et al., 2016).

\section{Orientación a la Administración e Internacionalización}

Miller, Le Breton-Miller, y Scholnick (2008) determinaron que la perspectiva de la administración puede tomar tres formas (a) administración sobre la continuidad, (b) administración sobre los empleados, (c) administración sobre los clientes. De acuerdo a Segaro et al. (2014) estos son los criterios que definen la orientación a la administración en la pequeña y mediana empresa familiar. La administración sobre la continuidad significa que los miembros de la empresa familiar están interesados en asegurar la longevidad de la compañía y por lo tanto en invertir en la creación de condiciones para lograr beneficios de largo plazo para los miembros de la familia (Sciascia et al., 2012). La administración sobre los empleados se refiere a la actitud para desarrollar a la fuerza de trabajo a través de la motivación y el entrenamiento, así como el transmitir un conjunto de valores (Davis et al., 1997; Sciascia et al., 2012). La administración sobre los clientes busca reforzar la relación con los clientes para mantener la prosperidad y supervivencia (Sciascia et al., 2012).

A pesar que no todas las empresas familiares poseen una orientación a la administración, cuanto ésta se encuentra presente puede originar ventajas competitivas gracias a sus miembros, su actitud colectiva, su compromiso psicosociológico y un comportamiento confiable, teniendo 
impacto positivo en el desempeño empresarial y por tanto en su internacionalización (Eddleston et al., 2012; Segaro et al., 2014). Basado en estos criterios, se postula la siguiente hipótesis:

H1: La orientación a la administración está positivamente relacionada con el grado de internacionalización.

La orientación a la administración del equipo gerencial mejora el desempeño de la empresa (Eddleston et al., 2012; Miller et al., 2008; Segaro, 2012). La flexibilidad estratégica constituye un elemento valioso para todas las empresas y resulta particularmente importante para la empresa familiar que con frecuencia ha sido considerada conservadora, lenta para reconocer y responder a los cambios del entorno y propensa a perder valiosas oportunidades que generaran beneficio y crecimiento (Zahra, Hayton, Neubaum, Dibrell, \& Craig, 2008). Las capacidades gerenciales de los miembros de la familia propietaria pueden llegar a convertirse en activos estratégicos para la empresa, los que aprovechados de manera adecuada permiten desarrollar la orientación a la administración en la empresa familiar mejorando su desempeño (Segaro, 2012).

Por tanto, la orientación a la administración es probable esté asociada a las capacidades del equipo gerencial y a su flexibilidad estratégica entre los cuales se incluyen a los miembros de la familia propietaria (Segaro et al., 2014). La flexibilidad estratégica del equipo gerencial permite que éstos tengan capacidad de reaccionar a las condiciones cambiantes del entorno, por lo que la mediana empresa familiar cuyo equipo gerencial posee una orientación a la administración, es estratégicamente flexible y aprovecha sus recursos, siendo más propensa a internacionalizarse (Segaro et al., 2014; Smith, Hair, \& Ferguson, 2014; Zahra et al., 2008). Basado en estos criterios, se propone la siguiente hipótesis:

H2: La orientación a la administración está positivamente relacionada con la flexibilidad estratégica del equipo gerencial

\section{Flexibilidad Estratégica del Equipo Gerencial e Internacionalización}

Un gran desafío para las empresas es desarrollarse en los actuales ambientes cambiantes y turbulentos, donde se ha incrementado la intensidad de los competidores, los ciclos de producto se han contraído, existen importantes cambios tecnológicos y una mayor globalización (Lin, 2012; Lin \& Liu, 2012; Nadkarni \& Herrmann, 2010). Dadas estas condiciones, es crucial para la empresa tener la capacidad de cambiar rápidamente de dirección y reconfigurarse estratégicamente para lograr éxito y una ventaja competitiva sostenible (Nadkarni \& Herrmann, 2010; Zahra et al., 2008). De ahí que las empresas necesiten lograr flexibilidad estratégica cuyo concepto ha tenido diversos significados en diferentes contextos, sin embargo, ésta puede comprenderse como la capacidad de reconocer amenazas y oportunidades, y aprovecharlas antes, durante y después de una crisis (Nadkarni \& Herrmann, 2010; Segaro et al., 2014). Se relaciona también con la capacidad de perseguir nuevas oportunidades, logrando responder de manera continua a cambios no anticipados y amenazas en el entorno competitivo de la empresa (Nadkarni \& Herrmann, 2010; Segaro et al., 2014; Zahra et al., 2008).

Este es un recurso valioso y particularmente importante para la empresa familiar, existiendo abundante evidencia empírica que muestra como en ambientes dinámicos, la 
flexibilidad estratégica de la empresa impulsa de forma positiva su desempeño, permitiéndole anticiparse a cambios ambientales, considerar de manera flexible diversas opciones, analizar alternativas y mecanismos para enfrentar la incertidumbre, e incluir además la capacidad de reversar decisiones estratégicas ineficientes y abandonar situaciones o relaciones equivocadas, incorporando acciones tanto ofensivas como defensivas, pudiendo ser éstas proactivas o reactivas (Lin, 2012; Merino, Monreal-Pérez, \& Sánchez-Marín, 2012; Nadkarni \& Herrmann, 2010; Peiris, Akoorie, \& Sinha, 2012; Zahra et al., 2008). El equipo gerencial de la empresa familiar necesita adoptar una estrategia flexible hacia la expansión internacional y tener la capacidad de tomar decisiones con una orientación internacional, si espera lograr y mantener la riqueza por medio de rendimientos potenciales de largo plazo (Lin, 2012; Nielsen \& Nielsen, 2011).

Estudios previos muestran una relación positiva entre las características del equipo gerencial y la internacionalización, por lo que un conocimiento heterogéneo en medio de una diversidad funcional del equipo gerencial ofrecería una visión más amplia del mundo, incluso en ambientes con menor incertidumbre (Agnihotri \& Bhattacharya, 2015; Nielsen \& Nielsen, 2011; Segaro et al., 2014). Basado en estos criterios, se propone la siguiente hipótesis:

H3: La flexibilidad estratégica del equipo gerencial está positivamente relacionada con el grado de internacionalización.

Las hipótesis propuestas se resumen en la Figura 1, donde se ilustra el marco conceptual.

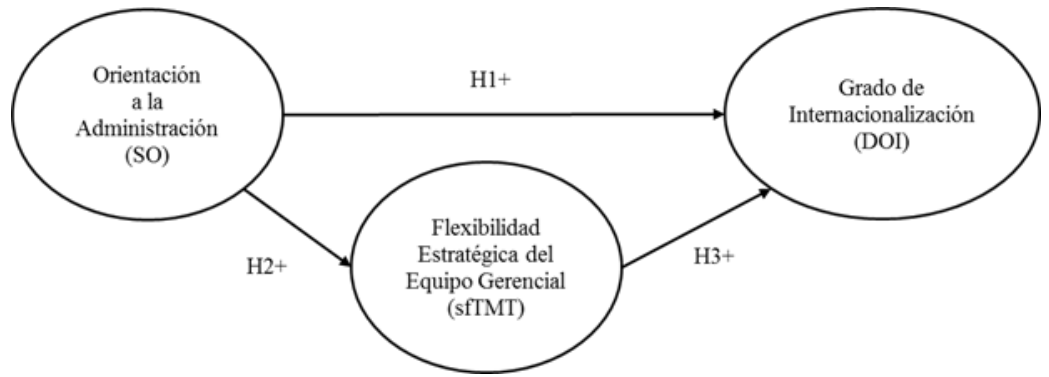

Figura 1. Relación propuesta entre la orientación a la administración y flexibilidad estratégica del equipo gerencial con el grado de internacionalización de la mediana empresa familiar.

\section{Método}

Para analizar las variables objeto de estudio se usó el método de mínimos cuadrado parciales [partial least squares] de ecuaciones estructurales (PLS-SEM) basado en varianza, mismo que resulta útil para el desarrollo y prueba de teorías complejas; y que prácticamente se ha convertido en un estándar en investigaciones en marketing y administración cuando se trata de analizar relaciones causa efecto entre constructos latentes (Hair, Ringle, \& Sarstedt, 2011, 2012; Nitzl, Roldan, \& Cepeda, 2016). El método PLS-SEM frecuentemente ofrece estimaciones más robustas para el modelo estructural, dado que se centra en el constructo endógeno del modelo, tiene la capacidad de realizar estimaciones de modelos muy complejos, incorpora constructos tanto formativos como reflectivos con pocas e incluso una variable de medida, permite el manejo flexible de variables moderadoras, relaciones no lineales o modelos jerárquicos, requiere tamaños pequeños de muestras, sin tener que asumir supuestos sobre la distribución de los datos, y posee la capacidad de procesar variables de escala nominal, ordinal, de intervalo y ratio (Hair 
et al., 2011; Hair, Sarstedt, Pieper, \& Ringle, 2012; Sarstedt, Ringle, \& Hair, 2014; Sarstedt, Ringle, Henseler, \& Hair, 2014; Sarstedt, Ringle, Smith, Reams, \& Hair, 2014).

Para explicar tanto el desempeño de la empresa familiar, como los resultados de su internacionalización, se ha utilizado la teoría de la administración, la cual sostiene que la empresa familiar muestra mayor actitud hacia el bienestar de largo plazo, incluyendo tanto sus empleados como clientes (Graves \& Thomas, 2008; Kontinen \& Ojala, 2012; Sciascia et al., 2012; Segaro et al., 2014). Estas características únicas, especialmente presentes en la mediana empresa familiar determinan su orientación a la administración (SO), la cual permite medir el gobierno de la empresa y que de acuerdo a Miller et al. (2008) se manifiesta y define en tres dimensiones: (a) orientación de largo plazo (LTO), relacionada a la continuidad del negocio; (b) orientación a los empleado (EMO), esto es el desarrollo continuo de una comunidad de empleados; y (c) orientación a los clientes (CMO), que hace referencia a mantener y desarrollar una cercana relación con los clientes (Sciascia et al., 2012; Segaro et al., 2014).

Dada la dinámica de los mercados internacionales, la flexibilidad estratégica resulta de gran importancia para la empresa y especialmente para la empresa familiar, porque ésta le permite evaluar y reconfigurar sus recursos y responder a las oportunidades o amenazas que se presenten (Lin, 2012). Equipos gerenciales heterogéneos es más probable que compensen sus limitaciones por medio de compartir su conocimiento y experiencia tanto en su mercado doméstico como en mercados internacionales (Johanson \& Vahlne, 1977, 2009). La aversión al riesgo puede verse compensada cuando miembros del equipo gerencial son diversos y poseen su propia red de relaciones (Calabrò \& Mussolino, 2013; Johanson \& Vahlne, 2009). Por tanto, la flexibilidad estratégica del equipo gerencial (sfTMT) constituye una variable que muestra el grado en que la empresa reacciona o modifica sus estrategias en respuesta a oportunidades o amenazas, y está dada por dos indicadores: (a) flexibilidad estratégica de la empresa (TSF), y (b) heterogeneidad del equipo gerencial (TMThe), los cuales son consistentes con los propuesto por Graves y Thomas (2006).

La variable endógena que mide la iniciativa empresarial internacional es el grado de internacionalización (DOI). Para medir el grado de internacionalización se consideraron tres indicadores reflectivos: (a) proporción de ventas en el exterior respecto a las ventas totales (FSTSR), (b) ámbito geográfico de las ventas (GESCPR), y (c) Porcentaje de empleados que dedican más del 50\% de su tiempo a actividades internacionales (EMPIA50) (Sullivan, 1994).

En la Figura 2 se presenta el diagrama de ruta [path diagram] para el modelo de ecuaciones estructurales propuesto donde constan las variables latentes tanto endógenas como exógenas, y sus indicadores reflectivos. 


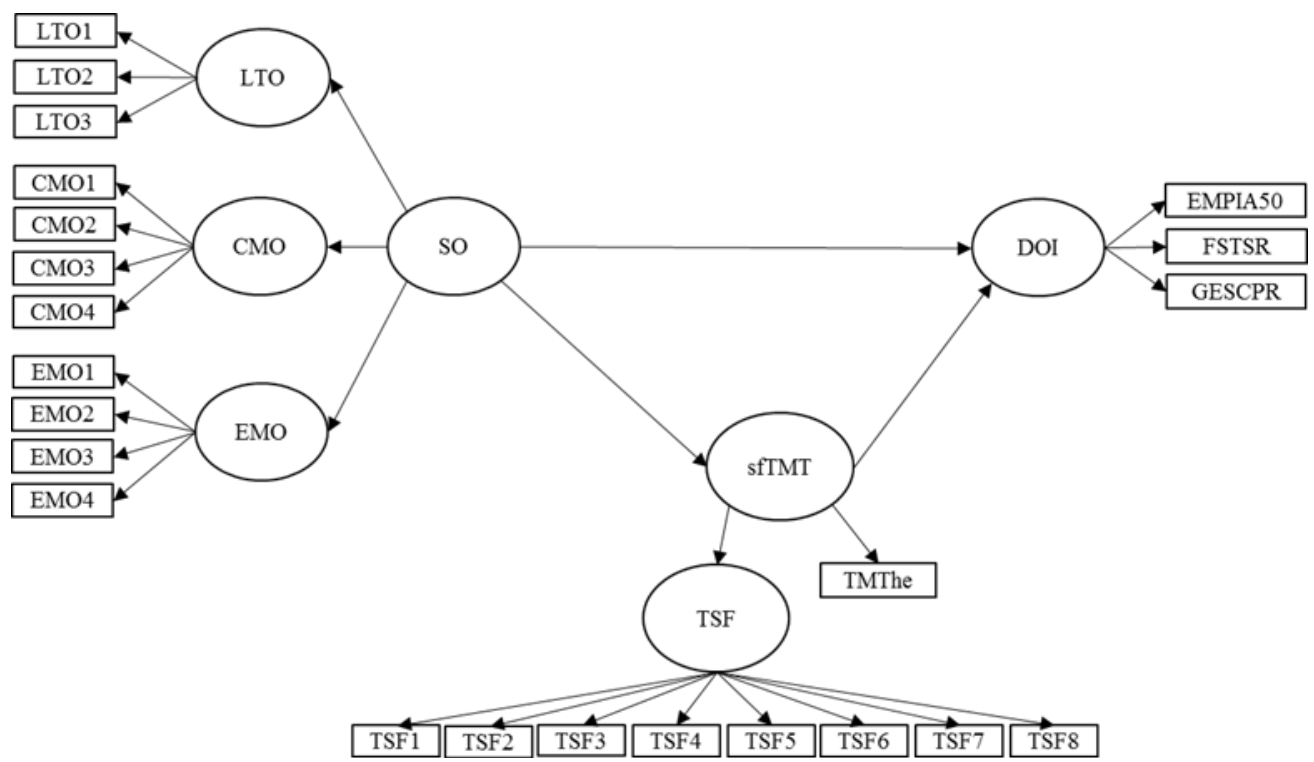

Figura 2. Diagrama de Ruta para la relación propuesta entre orientación a la administración, flexibilidad estratégica del equipo gerencial y grado de internacionalización para la mediana empresa familiar.

El instrumento utilizado fue la encuesta, misma que empleó Segaro et al. (2014) en una investigación en pequeñas y medianas empresas familiares en Finlandia, y que se derivó de estudios previos, por lo cual los indicadores de medida se basaron en escalas existentes siendo validado por diversos investigadores. Las tres dimensiones propuestas por Miller et al. (2008) fueron consideradas como indicadores que reflejan la orientación a la administración en la mediana empresa familiar. Las preguntas relacionadas a la orientación de largo plazo (LTO) fueron conceptualizadas por Segaro et al. (2014) desde el estudio realizado por Miller et al. (2008). La dimensión orientación a los empleado (EMO) fue tomada por Segaro et al. (2014) del estudio realizado por Zahra et al. (2008) donde una de las preguntas fue reemplazada. La dimensión de orientación a los clientes (CMO) fue tomada por Segaro et al. (2014) del estudio realizado por Reinartz, Krafft, y Hoyer (2004) donde se obtuvieron algunos resultados de la medición del proceso de implementación de sistemas de gestión de relaciones con clientes [customer relationship management]. Todas las preguntas se valoraron usando una escala Likert de cinco puntos. Para medir la flexibilidad estratégica del equipo gerencial (sfTMT) se usó un constructo conformado por dos indicadores: (a) la flexibilidad estratégica de la empresa (TSF) tomado del estudio realizado por Barringer y Bluedorn (1999), y (b) la diversidad funcional o heterogeneidad del equipo gerencial (TMThe) tomado del estudio realizado por Boeker y Wiltbank (2005). Para la flexibilidad estratégica de la empresa (TSF) de acuerdo a Segaro et al. (2014) en el cuestionario se introdujeron las preguntas 1, 2 y 3; y se usó una escala de Likert de cinco puntos. La heterogeneidad del equipo gerencial (TMThe), se espera incremente el nivel de flexibilidad en el equipo gerencial y se evaluó usando una sola pregunta donde se pidió seleccionar de múltiples opciones la experiencia de la alta gerencia, ésta se considera una variable dummy, por lo que la diversidad funcional total tendría una valoración entre 1 a 4 , donde 4 represente la mayor heterogeneidad y diferencia funcional (Segaro et al., 2014). Para el grado de internacionalización (DOI) las tres dimensiones propuestas por Sullivan (1994) fueron operacionalizadas en el estudio realizado por Segaro et al. (2014) considerando: (a) proporción de ventas en el exterior respecto a las ventas totales (FSTSR), (b) porcentaje de empleados que dedican más del $50 \%$ de su tiempo en actividades internacionales durante el presente año 
(EMPIA50), y (c) ámbito geográfico (GESCPR), mismo que considera la diversidad geográfica de las ventas internacionales, el cual fue medido preguntando si las ventas del último período fiscal se realizaron en su totalidad en la Comunidad Andina de Naciones o fuera de la misma en porcentajes entre el $1 \%$ al $24 \%, 25 \%$ al $49 \%$ o superior al $50 \%$. El ámbito geográfico se calculó como una ponderación simple para cada respuesta, asignando uno si todas las ventas se realizaron en la Comunidad Andina de Naciones, dos si éstas fueron entre el $1 \%$ al 24\%, tres si fueron entre el $25 \%$ al $49 \%$ y cuatro si fueron mayores al $50 \%$. Cada una de las tres dimensiones que mide el grado de internacionalización se convirtió en una variable de relación, pero se usaron de manera separada como tres indicadores reflectivos (Segaro et al., 2014; Yeoh, 2014).

El cuestionario estaba en idioma inglés por lo cual se tradujo al español, asegurando la misma. La validez de contenido se logró aplicando el mismo a dos empresas parte de la población objeto de estudio para posteriormente realizar entrevistas con los participantes y verificar su comprensión y relevancia de las variables observadas en el dominio del estudio. A los participantes se les indicó respondieran todas las preguntas de forma independiente y evitaran contestar sobre la situación futura, considerando únicamente la situación actual de la empresa; adicionalmente fue revisado por tres académicos conocedores tanto de empresas familiares como del método a aplicar en la presente investigación. Se solicitó y se obtuvo la correspondiente autorización por parte del autor para usar el instrumento aplicado. Se seleccionó a un funcionario por empresa para contestar la encuesta, quien además debía conocer sobre los planes y resultados de la internacionalización y ser el responsable de la misma. Con este mecanismo se buscó alcanzar una tasa de participación elevada, homogeneidad en las personas que respondieron la encuesta, asegurando la confidencialidad en las respuestas individuales y cuyos participantes fueron informados del objetivo de la investigación, dando su consentimiento. Por ello, los resultados del presente estudio únicamente muestran información agregada y no respuestas específicas o individuales de cada cuestionario.

\section{Población y Muestra}

La población objeto de estudio está constituida por medianas empresas domiciliadas en Ecuador, que tienen carácter familiar. La unidad de análisis es la empresa. Por conveniencia en la toma de información de campo, se ha considerado en el estudio, las empresas de los sectores florícola y agro industrial (Instituto Nacional de Estadística y Censos [INEC], 2014) por constituir sectores similares y ser predominantemente exportadores. De acuerdo a la información publicada por el Directorio de Empresas y Establecimientos 2013 (Instituto Nacional de Estadística y Censos [INEC], 2013), en el Ecuador existen un total de 810,272 empresas, de las cuales 343 constituyen medianas empresas de acuerdo a la definición dada por la Comunidad Andina de Nacional, llevan registros contables, están activas, realizan actividades de elaboración y exportación de bienes y correspondan al sector privado (Comunidad Andina, 2009; INEC, 2013) por "pertenecer a un agente económica que no está relacionado por el Estado" (Pino, 2008, p. 98). La población objeto de estudio no pudo ser completamente definida, dado que no existe una base de datos que clasifique las empresas como familiares o no, aunque se puede asumir la mayoría de las medianas empresa lo son (Hiebl, 2013; IFERA, 2003; Kontinen \& Ojala, 2012), por lo cual se decidió usar una muestra por conveniencia no probabilística. La unidad de análisis fue la empresa. 


\section{Recolección y Análisis de Datos}

El Instituto de Promoción de Exportaciones e Inversiones del Ecuador (PRO ECUADOR) facilitó la base de datos de las empresas de los sectores florícola y agro industrial, por lo cual se procedió a contactar de manera personal o telefónica a un funcionario por empresa, que debía conocer sobre los planes y resultados de la internacionalización y ser responsable de la misma, para solicitar complete la encuesta una vez informado respecto a los objetivos de la investigación y que haya manifestado su consentimiento para participar voluntariamente, asegurando el anonimato y confidencialidad de sus respuestas. En lo casos que alguna de las empresas solicitó completar el formulario de electrónica, éste fue preparado en formato digital permitiendo sean llenados directamente, facilitando y reforzando su recolección.

Inicialmente los datos fueron analizados para verificar que los cuestionarios completados cumplan con lo definido en la población objeto, para posteriormente usando el software SPSS $®$ versión 21.0 explorar los mismos y finalmente usado el software WarpPLS versión 5.0 obtener y analizar los resultados del modelo de ecuaciones estructurales propuesto.

\section{Resultados}

Del sector florícola se recolectaron un total de 114 cuestionarios y del sector agro industrial un total de 127 cuestionarios, sin embargo, luego de revisar los formularios recibidos y verificar si éstos se ajustan a la población objeto de estudio, si estaban correctamente llenados y completos, se aceptaron 75 casos para empresas del sector florícola y 42 casos para empresas del sector agro industrial, lo cual representa un total de 117 casos válidos, mismos que respecto a la población constituyen un $34.1 \%$, porcentaje considerado satisfactorio dentro de investigaciones de esta naturaleza (e.g., Berent-Braun \& Uhlaner, 2010; Calabrò \& Mussolino, 2013; Miller et al., 2008; Segaro et al., 2014; Zahra et al., 2008). De acuerdo a Miller et al. (2008) las tasas de respuesta que normalmente se obtienen en investigaciones que utilizan encuestas fluctúan entre el $25 \%$ al $35 \%$. Los resultados para las respuestas de cada uno de los indicadores reflectivos que representan las variables exógenas se muestran en la Tabla A1, Tabla A2 y Tabla A3. Para el caso del variable endógeno grado de internacionalización, los resultados se resumen en la Tabla A4.

Con los 117 casos válidos se realizaron varias pruebas para identificar: (a) datos perdidos, (b) valores extremos y casos atípicos, (c) normalidad de datos, y (d) se realizó una prueba de diferencia de medias para los dos sectores considerados en el estudio. No se encontraron datos perdidos, por lo cual no fue necesario desarrollar ningún método para la imputación de los mismos. En segundo lugar se analizaron los valores extremos y casos atípicos cuyo objetivo es "encontrar inconsistencias en el llenado de la encuesta por parte de los participantes" (Pino, 2008, p. 133). Para el indicador EMO1 se detectaron cuatro casos con valores extremos, para el indicador EMO2 y COM4 se detectaron tres casos con valores extremos. Los indicadores TSF2, TSF3, TSF4 reportaron cinco casos extremos y el indicador TSF7 dos casos extremos. En total fueron 17 encuestas que presentaron al menos un dato con valor extremo en una o más preguntas, por lo que estas fueron analizadas sin que se encuentren patrones de respuesta o motivos por los cuales puedan considerarse mal llenadas, manteniéndose como válidas y considerando la totalidad en el análisis. Se realizó además el procedimiento de detección de 
anomalías por medio de la identificación de casos atípicos para la totalidad de indicadores reflectivos, sin que se llegue a determinar la presencia de anomalía. En tercer lugar, se realizó una prueba de normalidad de datos aplicando las pruebas de Kolmogorov-Smirnov y ShapiroWilk con un intervalo de confianza del $95 \%$, llegando a obtener resultados donde todas las variables muestran niveles de significancia menores a .05, por lo cual se puede concluir que los datos no siguen una distribución normal. Una de las características del modelo PLS-SEM, es su capacidad de realizar estimaciones de modelos muy complejos sin tener que asumir supuestos sobre la distribución de los datos, característica que no es aceptada por el modelo de ecuaciones estructurales basado en covarianza (Hair, Sarstedt, et al., 2012; Sarstedt, Ringle, \& Hair, 2014; Sarstedt, Ringle, Henseler, et al., 2014). Este resultado ratifica la necesidad de utilizar el método PLS-SEM para la presente investigación. Finalmente se realizó una prueba de diferencia de medias, aplicando la prueba $\mathrm{Z}$ de Kolmogorov-Smirnov, misma que se utiliza para determinar si dos muestras independientes provienen de una misma población, llegando a obtenerse para todos los indicadores un nivel de significancia mayor a .05, por lo cual se concluyó que éstas provienen de una misma población para los dos sectores considerados. Con este resultado los datos fueron integrados.

\section{Tamaño de la Muestra}

Una de las características del método PLS-SEM es su capacidad de trabajar con tamaños de muestra pequeños, ofreciendo capacidades de muestreo que no brindan otras herramientas de análisis multivariante, mostrándose robusto y relativamente con una alta capacidad estadística, sin que esto signifique que sea un método exento de un adecuado tamaño de muestra (Hair, Ringle, \& Sarstedt, 2013; Sarstedt, Ringle, \& Hair, 2014; Sarstedt, Ringle, Smith, et al., 2014). Hair, Sarstedt, et al. (2012) recomendaron utilizar la regla de las diez veces [ten time rules] como mecanismo de estimación aproximada para determinar el tamaño de la muestra. Esta regla, para modelos con indicadores reflectivos es equivalente a utilizar un tamaño de muestra diez veces el mayor número de rutas o caminos estructurales que se dirigen a un constructo latente particular en el modelo estructural o modelo interior (Hair, Sarstedt, et al., 2012; Sarstedt, Ringle, Smith, et al., 2014). Sarstedt, Ringle, y Hair (2014) recomendaron que el mínimo número de observaciones es un rango entre 30 y 100 casos. El tamaño obtenido de muestra en el presente estudio es 117 casos, mismo que supera lo indicado, considerándose por tanto adecuado.

\section{Resultados del Modelo}

El método PLS-SEM requiere completar dos instancias: (a) evaluar el modelo de medida [measurement model], y (b) evaluar el modelo estructural [structural model] (Hair et al., 2011; Sarstedt, Ringle, Smith, et al., 2014).

\section{Evaluación del Modelo de Medida}

El modelo propuesto en la presente investigación considera únicamente constructos de medida reflectivos, por lo cual, para establecer su confiabilidad y validez, se analizaron los resultados de: (a) indicador de confiabilidad [indicator reliability] llamado también indicador de carga [indicator loadings], (b) confiabilidad de la consistencia interna [internal consistency reliability] del constructo, mismo que se puede lograr evaluando la confiabilidad compuesta 
[composite reliability]; (c) validez convergente [convergent validity], y (d) validez discriminante [discriminant validity] del modelo de medida (Sarstedt, Ringle, Smith, et al., 2014).

El indicador de confiabilidad se obtiene a partir de la tabla de cargas combinadas y cargas transversales [combined loadings and cross-loadings] y donde los valores de carga de las variables latentes reflectivas deben ser mayores o iguales a 0.4 , valores menores deben ser retirados del modelo y los valores de carga transversal deben ser menores (Hair et al., 2011; Kock, 2015). El nivel de significancia (p-value) para todos los indicadores reflectivos se considera un parámetro de validación del análisis factorial confirmatorio, esperando valores menores a .05 (Kock, 2015). En la Tabla 1 se presentan los resultados evidenciando que se cumple con lo especificado, por lo que ninguna variable o indicador fue retirado de modelo.

Tabla 1. Indicadores de Carga Combinada y Carga Transversal

\begin{tabular}{cccccc}
\hline & DOI & SO & TMT & Tipo & P value \\
\hline EMPIA50 & $(0.591)$ & 0.189 & -0.156 & Reflectivo & $<0.001$ \\
\hline FSTSR & $(0.878)$ & -0.108 & 0.146 & Reflectivo & $<0.001$ \\
\hline GESCPR & $(0.528)$ & -0.053 & -0.163 & Reflectivo & $<0.001$ \\
\hline LTO & -0.174 & $(0.402)$ & -0.093 & Reflectivo & $<0.001$ \\
\hline EMO & 0.070 & $(0.841)$ & -0.158 & Reflectivo & $<0.001$ \\
\hline CMO & -0.009 & $(0.884)$ & 0.159 & Reflectivo & $<0.001$ \\
\hline TSF & -0.046 & 0.172 & $(0.972)$ & Reflectivo & $<0.001$ \\
\hline TMThe & 0.178 & -0.663 & $(0.414)$ & Reflectivo & $<0.001$ \\
\hline
\end{tabular}

La confiabilidad de la consistencia interna del constructo, se evalúo con la confiabilidad compuesta siendo este criterio más adecuado en modelos de ecuaciones estructurales basado en varianza (Hair et al., 2011; Kock, 2015; Sarstedt, Ringle, Smith, et al., 2014). El coeficiente de confiabilidad compuesta constituye una medida de la confiabilidad asociada con las variables latentes y una medida de confiabilidad de mayor aceptación (Hair et al., 2011; Kock, 2015). De acuerdo a Sarstedt, Ringle, Smith, et al. (2014) valores de confiabilidad compuesta entre $0.60 \mathrm{y}$ 0.70 se consideran aceptables. En la Tabla 2 se presentan los resultados de confiabilidad compuesta siendo todos superiores a 0.6 , cumpliendo lo requerido. La validez convergente fue evaluada por la varianza media extraída [average variance extracted] (AVE) para todos los ítems asociados con cada constructo (Sarstedt, Ringle, Smith, et al., 2014). En la Tabla 2 se presentan los resultados obtenido para AVE y donde de acuerdo a Kock (2015) valores son superiores a 0.5 resultan aceptables, indicando que en promedio, el constructo explica sobre el $50 \%$ de la varianza de sus ítems (Sarstedt, Ringle, Smith, et al., 2014).

Tabla 2. Resultados de los coeficientes de las variables latentes

\begin{tabular}{lccc}
\hline & DOI & SO & sfTMT \\
\hline $\begin{array}{l}\text { Índice de } \\
\text { confiabilidad } \\
\text { compuesta }\end{array}$ & 0.713 & 0.770 & 0.685 \\
$\begin{array}{l}\text { Varianza Media } \\
\text { Extraída (AVE) }\end{array}$ & 0.566 & 0.550 & 0.558 \\
\hline
\end{tabular}


La varianza media extraída (AVE) es igualmente usada para evaluar la validez discriminante (Kock, 2015; Sarstedt, Ringle, Smith, et al., 2014) y cuyos resultados se usan conjuntamente con la correlación de las variables latentes (Kock, 2015). En la Tabla 3 se presentan los resultados de la correlación entre las variables latentes con la raíz cuadrada de AVE [correlations among latent variables with square roots of AVEs]. De acuerdo a Kock (2015) la validez discriminante se evalúa considerando que los valores de la diagonal de la Tabla 3 deben ser mayores que cualquier otro valor por encima o por debajo de ellos en la misma columna, o deben ser superiores a cualquier otro valor de su izquierda o derecha en la misma fila. Como se aprecia los resultados cumplen con lo especificado logrando el modelo de medida validez discriminante.

Tabla 3. Correlación entre Variables Latentes con las Raíces Cuadradas de AVEs

\begin{tabular}{lccc}
\hline & DOI & SO & sfTMT \\
\hline DOI & 0.683 & 0.308 & 0.291 \\
SO & 0.308 & 0.742 & 0.599 \\
sfTMT & 0.291 & 0.599 & 0.747 \\
\hline
\end{tabular}

Nota. Valores de las raíces cuadradas de AVE se muestran en la diagonal

\section{Evaluación del Modelo Estructural}

El objetivo del modelo de ecuaciones estructurales usando el método de mínimos cuadrados parciales, es explicar la varianza de las variables dependientes cuando se examina el modelo (Sarstedt, Ringle, Smith, et al., 2014). A diferencia del modelo de ecuaciones estructurales basado en covarianza, el modelo de ecuaciones estructurales de mínimos cuadrados parciales basado en varianza no posee un estándar estadístico de bondad de ajuste y en su lugar la evaluación de la calidad del modelo se sustenta en su capacidad de predecir el constructo endógeno (Hair et al., 2013; Kock, 2015; Sarstedt, Ringle, Smith, et al., 2014). Los criterios de evaluación utilizados son: (a) evaluación del modelo estructural de potencial colinealidad entre constructos predictores, (b) evaluación del coeficiente de determinación (R2) como indicador de la capacidad predictiva del modelo, (c) evaluación del índice de redundancia de validez cruzada [cross-validated redundancy] (Q2) conocido como indicador de Stones-Geisser, el cual permite evaluar la relevancia o capacidad predictiva del modelo; y (d) la significancia y relevancia de los coeficientes de ruta [path coefficients] (Sarstedt, Ringle, Henseler, et al., 2014; Sarstedt, Ringle, Smith, et al., 2014).

La colinealidad se evaluó por el índice de colinealidad completa o también llamado multicolinealidad de los factores de inflación de la varianza [full collinearity variance inflation factors] (VIFs). Los resultados obtenidos se presentan en la Tabla 4 y como regla general un resultado menor o igual a 3.3, sugiere la no existencia de multicolinealidad en el modelo (Kock, 2015). El coeficiente R2 constituye una medida de la exactitud predictiva del modelo en términos de predicción dentro de la muestra (Hair, Sarstedt, et al., 2012; Kock, 2015; Sarstedt, Ringle, Smith, et al., 2014). El coeficiente Q2 constituye otra forma de evaluar la relevancia predictiva del modelo y es considerado una medida de predicción fuera o independiente de la muestra, y una medida tradicional no paramétrica utilizada para evaluar la capacidad predictiva asociada con cada bloque de variables latentes en el modelo a través de la variable latente 
endógena que es la variable criterio en el bloque (Hair et al., 2011; Kock, 2015; Sarstedt, Ringle, Henseler, et al., 2014; Sarstedt, Ringle, Smith, et al., 2014). Lo resultados de los coeficientes R2 y Q2 se presentan en la Tabla 4 donde se obtienen coeficientes mayores a cero por lo que se evidencia la validez predictiva del modelo en relación con la variable latente endógena (Kock, 2015).

Tabla 4. Resultados de coeficientes para el modelo estructural

\begin{tabular}{lccc}
\hline & DOI & SO & sfTMT \\
\hline $\begin{array}{l}\text { Colinealidad } \\
\text { completa VIF }\end{array}$ & 1.127 & 1.608 & 1.591 \\
Coeficiente & 0.147 & & 0.364 \\
$\mathrm{R}^{2}$ & & \\
$\begin{array}{l}\text { Coeficiente } \\
\mathrm{Q}^{2}\end{array}$ & 0.168 & & 0.372 \\
\hline
\end{tabular}

Los coeficientes de ruta $(\beta)$ se interpretan como coeficientes beta estandarizados de regresión de mínimos cuadrados ordinarios (Hair et al., 2011). Resultados de coeficiente de ruta no significativos o que muestren un signo contrario a las hipótesis plateadas, significa que no soporta las mismas, mientras que resultados del coeficiente de ruta que muestran la misma dirección de las hipótesis, significa que empíricamente apoyan la relación causal propuesta (Hair et al., 2013; Kock, 2015; Sarstedt, Ringle, Smith, et al., 2014; Smith et al., 2014). Los resultados de coeficientes de ruta $(\beta)$ y su nivel de significancia para el modelo estructural propuesto se muestran en la Tabla 5, donde todos los valores del coeficiente de ruta son mayores a 0.02 y estadísticamente significativos $(\mathrm{p}<.05)$ permitiendo con los mismos concluir sobre las hipótesis propuestas.

Tabla 5. Coeficientes de Ruta ( $\beta$ ) y Nivel de Significancia del Modelo Estructural

\begin{tabular}{|c|c|c|c|}
\hline & DOI & SO & sfTMT \\
\hline \multirow{2}{*}{ DOI } & & 0.275 & 0.156 \\
\hline & & $(p<.001)$ & $(p=.041)$ \\
\hline \multicolumn{4}{|l|}{ SO } \\
\hline \multirow{2}{*}{ sfTMT } & & 0.604 & \\
\hline & & $(p<.001)$ & \\
\hline
\end{tabular}

La hipótesis $\mathrm{H} 1$ propone una relación positiva entre la orientación a la administración (SO) y el grado de internacionalización (DOI). El resultado obtenido soporta la hipótesis H1, pues el coeficiente de ruta $(\beta=0.275)$ es positivo y estadísticamente significativo $(\mathrm{p}<.001)$, siendo consistente con la literatura, y evidenciando como la teoría de la administración aplicada a la empresa familiar sostiene que los administradores poseen un alto sentido del deber hacia la empresa y buscan el bienestar colectivo y no individual, mejorando tanto su desempeño como los resultados de su internacionalización (e.g., Choi, Zahra, Yoshikawa, \& Han, 2015; Graves \& 
Thomas, 2008; Miller et al., 2008; Sciascia et al., 2012; Segaro et al., 2014). La hipótesis H1 fue aceptada. La presente investigación considera la orientación a la administración y su influencia en la internacionalización en la mediana empresa familiar, acoplada con la flexibilidad estratégica del equipo gerencial (Miller et al., 2008; Segaro et al., 2014). La orientación a la administración al estar presente en la empresa familiar, sustenta el bienestar de largo plazo de la empresa, sus clientes y empleados, mientras que la flexibilidad estratégica del equipo gerencial para la empresa familiar es particularmente valioso e importante, ya que le ofrece la capacidad de adaptarse a circunstancias de cambio y atender la demanda de sus clientes, adoptar tecnologías emergentes y desarrollar nuevos mercados (Graves \& Thomas, 2006; Lin, 2012; Segaro et al., 2014). Es por esto que la orientación a la administración acoplada con la flexibilidad estratégica del equipo gerencial, entre los que se incluyen a miembros de la familia le permitirá a la empresa buscar oportunidades en mercados externos, aportando positivamente a la internacionalización (Segaro et al., 2014; Zahra et al., 2008). La hipótesis H2 considera una relación positiva entre la orientación a la administración y la flexibilidad estratégica del equipo gerencial, y la hipótesis H3 plantea una relación positiva entre la flexibilidad estratégica del equipo gerencial y el grado de internacionalización. El coeficiente de ruta entre la orientación a la administración (SO) y la flexibilidad estratégica del equipo gerencial (sfTMT) es positivo $(\beta=0.604)$ y estadísticamente significativo $(\mathrm{p}<.001)$ por lo cual la hipótesis $\mathrm{H} 2$ fue aceptada, así mismo el coeficiente de ruta entre la flexibilidad estratégica del equipo gerencial (sfTMT) y el grado de internacionalización (DOI) fue positivo $(\beta=0.156)$ y estadísticamente significativo $(\mathrm{p}=.041)$ por lo cual la hipótesis H3 fue aceptada. Los resultados muestran que la orientación a la administración, acoplada con la flexibilidad estratégica del equipo gerencial influye de manera positiva en la internacionalización, siendo este resultado consistente con lo encontrado en la revisión de la literatura.

\section{Discusión}

Los sistemas de la familia y la empresa comparten supuestos y valores, e inciden en la cultura, estrategia e internacionalización de la empresa familiar. La orientación a la administración se relaciona de manera positiva y significativa con el grado de internacionalización. Este resultado puede comprenderse dado que la orientación a la administración, la cual está siempre presente en la empresa familiar, le permite crear y desarrollar ventajas competitivas gracias a sus miembros, su actitud colectiva, su compromiso psicosociológico y comportamiento confiable, teniendo un impacto positivo en su desempeño e internacionalización. Es importante comprender el rol que tienen tres dimensiones que conforman la orientación a la administración: orientación de largo plazo, orientación a los empleados y orientación a los clientes. La orientación de largo plazo permite a la empresa familiar mantener de manera sustentable el negocio a lo largo de las generaciones, la orientación a los empleados le permite crear redes de negocio como un mecanismo que facilita su internacionalización, siendo estas redes fundamentales para el reconocimiento de oportunidades en mercados externos; y la orientación a los clientes le permite mantener su prosperidad y supervivencia. Si bien estas tres dimensiones constituyen factores relacionados al capital social e internacionalización de la empresa familiar, es la orientación de largo plazo la que fomenta su desarrollo en el equipo gerencial. 
La orientación a la administración muestra una relación positiva y significativa con la flexibilidad estratégica del equipo gerencial. La flexibilidad estratégica del equipo gerencial resulta un elemento valioso y particularmente importante para la empresa familiar. Es la orientación a la administración presente en equipos gerenciales flexibles, la que permite a la empresa reconocer y responder a cambios del entorno, así como identificar y aprovechar oportunidades que generen beneficio y crecimiento para la misma. La orientación a la administración acoplada con la flexibilidad estratégica del equipo gerencial aporta de manera positiva a la internacionalización. Por tanto, el grado de participación internacional de la empresa es el reflejo de la capacidad y entusiasmo de su equipo gerencial.

\section{Implicaciones}

La presente investigación tiene relevancia para propietarios, administradores, consultores, así como para académicos que estudian la internacionalización de la empresa familiar y los factores que la motivan. Los propietarios que participan o no en su administración y buscan la supervivencia de su empresa a lo largo de las generaciones, deben tener presente que la orientación a la administración constituye un mecanismo por el cual los administradores logran un alto sentido del deber hacia la organización, protegiendo y maximizando la riqueza del accionista, buscando el bienestar colectivo antes que individual, impactando positivamente en su desempeño y expansión internacional.

La falta de especialistas en mercados internacionales en el equipo gerencial constituye una barrera para la internacionalización y en caso de no contar con estos especialistas entre los miembros de la familia, la empresa debe estar dispuesta a recibir miembros no familiares que le permitan mejorar su capacidad para responder a los retos empresariales. Por tanto, la empresa familiar que busca expandirse en mercados externos necesita desarrollar su orientación a la administración y contar con equipos gerenciales con flexibilidad estratégica, lo cual le permitirá mirar el entorno, las condiciones cambiantes, y ajustar su estrategia de negocio, manteniendo una visión de largo plazo.

En cuanto a las implicaciones en el mundo académico, la presente investigación aporta conocimiento sobre internacionalización de la empresa familiar y los factores que la motivan. En Ecuador no se han identificado estudios de esta naturaleza por lo cual se busca contribuir con evidencia empírica para el país.

\section{Recomendaciones}

Futuros estudios podrían considerar las capacidades gerenciales necesarias para la internacionalización en la mediana empresa familiar conjuntamente con la orientación a la administración como elementos relacionados. En relación al equipo gerencial, futuros estudios podrían considerar su composición, incluyendo la participación de la familia propietaria y su estadío generacional, así como sus características demográficas, estudiando el rol del equipo gerencial en la internacionalización e identificando las diferencias entre empresas familiares que se internacionalizan en la primera o siguientes generaciones, permitiendo así comprender el rol del estadío generacional. 
La presente investigación considera el sector florícola y agro industrial. Se recomienda profundizar e incorporar a otros sectores económicos, considerando la posibilidad de incluir sectores exportadores tanto de bienes como de servicios, determinando si los factores de internacionalización considerados en el presente estudio tienen el mismo comportamiento.

\section{Bibliografía}

Abdellatif, M., Amann, B., \& Jaussaud, J. (2010). Family versus nonfamily business: A comparison of international strategies. Journal of Family Business Strategy, 1(2), 108-116. doi: 10.1016/j.jfbs.2010.04.004

Acedo, F. J., Casillas, J. C., \& Moreno, A. M. (2008). La internacionalizacion de la empresa familiar: Un análisis exploratorio. Management International, 12(3), 77-123.

Agnihotri, A., \& Bhattacharya, S. (2015). Determinants of export intensity in emerging markets: An upper echelon perspective. Journal of World Business, 50(4), 687-695. doi: 10.1016/j.jwb.2014.11.001

Alkaabi, S. K., \& Dixon, C. (2014). Factors affecting internationalization decision making in family businesses: An integrated literature review. Journal of Applied Management and Entrepreneurship, 19(2), 53-77.

Barringer, B. R., \& Bluedorn, A. C. (1999). The relationshio between corporate entrepreneurship and strategic management. Strategic Management Journal, 20(5), 421-444. doi: 10.1002/(SICI)10970266(199905)20:5<421::AID-SMJ30>3.0.CO;2-O

Berent-Braun, M. M., \& Uhlaner, L. M. (2010). Family governance practices and teambuilding: paradox of the enterprising family. Journal of Small Business Economics, 38(1), 103-119. doi: $10.1007 / \mathrm{s} 11187-010-9269-4$

Boeker, W., \& Wiltbank, R. (2005). New venture evolution and managerial capabilities. Organization Science, 16(2), 123-133. doi: 10.i287/orsc.1050.0115

Calabrò, A., \& Mussolino, D. (2013). How do boards of directors contribute to family SME export intensity? The role of formal and informal governance mechanisms. Journal of Management \& Governance, 17(2), 363-403. doi: 10.1007/s10997-011-9180-7

Cerrato, D., \& Piva, M. (2012). The internationalization of small and medium-sized enterprises: the effect of family management, human capital and foreign ownership. Journal of Management \& Governance, 16(4), 617-644. doi: 10.1007/s10997-010-9166-x

Comunidad Andina. (2009). Resolución 1260. Disposición Técnica para la Transmisión de Datos de Estadística de PYME de los Países Miembros de la Comunidad Andina. Lima-Perú: Secretaría General de la Comunidad Andina. Recuperado de http://www.comunidadandina.org/Seccion.aspx?id=79\&tipo=TE\&title=pymes.

Craig, J. B., Dibrell, C., \& Garrett, R. (2014). Examining relationships among family influence, family culture, flexible planning systems, innovativeness and firm performance. Journal of Family Business Strategy, 5(3), 229-238. doi: 10.1016/j.jfbs.2013.09.002 
Choi, Y. R., Zahra, S. A., Yoshikawa, T., \& Han, B. H. (2015). Family ownership and R\&D investment: The role of growth opportunities and business group membership. Journal of Business Research, 68(5), 1053-1061. doi: 10.1016/j.jbusres.2014.10.007

Chrisman, J. J., Chua, J. H., \& Sharma, P. (2005). Trends and directions in the development of a strategic management theory of the family firm. Entrepreneurship Theory and Practice, 29(5), 555-575. doi: 10.1111/j.1540-6520.2005.00098.x

Davis, J. H., Schoorman, F. D., \& Donaldson, L. (1997). Toward a Stewardship Theory of management. Academy of Management Review, 22(1), 20-47. doi: 10.5465/AMR.1997.9707180258

Eddleston, K. A., Kellermanns, F. W., \& Zellweger, T. M. (2012). Exploring the entrepreneurial behavior of family firms: Does the Stewardship Perspective explain differences? Entrepreneurship Theory and Practice, 36(2), 347-367. doi: 10.1111/j.1540-6520.2010.00402.x

Fernández, Z., \& Nieto, M. J. (2005). Internationalization strategy of small and medium-sized family businesses: Some influential factors. Family Business Review, 18(1), 77-89. doi: 10.1111/j.17416248.2005.00031.x

Garcia-Castro, R., \& Aguilera, R. V. (2014). Family involvement in business and financial performance: A set-theoretic cross-national inquiry. Journal of Family Business Strategy, 5(1), 85-96. doi: 10.1016/j.jfbs.2014.01.006

Graves, C., \& Thomas, J. (2006). Internationalization of australian family businesses: A managerial capabilities perspective. Family Business Review, 19(3), 207-224. doi: 10.1111/j.17416248.2006.00066.x

Graves, C., \& Thomas, J. (2008). Determinants of the internationalization pathways of family firms: An examination of family influence. Family Business Review, 21(2), 151-167. doi: 10.1111/j.17416248.2008.00119.x

Hair, J. F., Ringle, C. M., \& Sarstedt, M. (2011). PLS-SEM: Indeed a Silver Bullet. The Journal of Marketing Theory and Practice, 19(2), 139-152. doi: 10.2753/mtp1069-6679190202

Hair, J. F., Ringle, C. M., \& Sarstedt, M. (2012). Partial Least Squares: The Better Approach to Structural Equation Modeling? Long Range Planning, 45(5-6), 312-319. doi: 10.1016/j.lrp.2012.09.011

Hair, J. F., Ringle, C. M., \& Sarstedt, M. (2013). Partial Least Squares Structural Equation Modeling: Rigorous Applications, Better Results and Higher Acceptance. Long Range Planning, 46(1-2), 112. doi: 10.1016/j.lrp.2013.01.001

Hair, J. F., Sarstedt, M., Pieper, T. M., \& Ringle, C. M. (2012). The Use of Partial Least Squares Structural Equation Modeling in Strategic Management Research: A Review of Past Practices and Recommendations for Future Applications. Long Range Planning, 45(5-6), 320-340. doi: 10.1016/j.lrp.2012.09.008

Hiebl, M. R. W. (2013). Management accounting in the family business: tipping the balance for survival. Journal of Business Strategy, 34(6), 19-25. doi: 10.1108/jbs-07-2013-0052

IFERA. (2003). Family businesses dominate: International Family Enterprise Reseach Academy. Family Business Review, 16(4), 235-240. doi: 10.1177/08944865030160040201 
INEC. (2013). Directorio de Empresas 2013 [Empresas_2013.sav]. Recuperado de http://www.ecuadorencifras.gob.ec/directoriodeempresas/

INEC. (2014). Compendio Estadístico 2014. Quito-Ecuador: Instituto Nacional de Estadística y Censos. Recuperado de http://www.ecuadorencifras.gob.ec/directoriodeempresas/.

Johanson, J., \& Vahlne, J.-E. (1977). The internationalization process of the firm-A model of knowledge development and increasing foreign market commitments. Journal of International Business Studies, 8(1), 23-32. doi: 10.1057/palgrave.jibs.8490676

Johanson, J., \& Vahlne, J.-E. (2009). The Uppsala internationalization process model revisited: From liability of foreignness to liability of outsidership. Journal of International Business Studies, 40(9), 1411-1431. doi: 10.1057/jibs.2009.24

Kock, N. (2015). WarpPLS 5.0 User Manual. Laredo, TX: ScriptWarp System.

Kontinen, T., \& Ojala, A. (2010). The internationalization of family businesses: A review of extant research. Journal of Family Business Strategy, 1(2), 97-107. doi: 10.1016/j.jfbs.2010.04.001

Kontinen, T., \& Ojala, A. (2011). International Opportunity Recognition among Small and Medium-Sized Family Firms. Journal of Small Business Management, 49(3), 490-514. doi: 10.1111/j.1540627X.2011.00326.X

Kontinen, T., \& Ojala, A. (2012). Internationalization pathways among family-owned SMEs. International Marketing Review, 29(5), 496-518. doi: 10.1108/02651331211260359

Le, V., \& Valadkhani, A. (2014). Are exporting manufacturing SMEs more efficient than non-exporting ones? Evidence from Australia's business longitudinal database. Economic Analysis and Policy, 44(3), 310-217. doi: 10.1016/j.eap.2014.07.002

Lin, W.-T. (2012). Family ownership and internationalization processes: Internationalization pace, internationalization scope, and internationalization rhythm. European Management Journal, 30(1), 47-56. doi: 10.1016/j.emj.2011.10.003

Lin, W.-T., \& Liu, Y. (2012). Successor characteristics, change in the degree of firm internationalization and firm performance: The moderating role of environmental uncertainty. Journal of Management \& Organization, 18(1), 16-35. doi: 10.1017/S1833367200001048

Madison, K., Holt, D. T., Kellermanns, F. W., \& Ranft, A. L. (2016). Viewing Family Firm Behavior and Governance through the Lens of Agency and Stewardship Theories. Family Business Review, 29(1), 65-93. doi: 10.1177/0894486515594292

Merino, F., Monreal-Pérez, J., \& Sánchez-Marín, G. (2012, 25-May-2012). Family firm internationalization: Influence of familiness on the Spanish firm export activity. Kiel Institute for the World Economy, 1-33. Recuperado de http://hdl.handle.net/10419/60329.

Miller, D., Le Breton-Miller, I., \& Scholnick, B. (2008). Stewardship vs. Stagnation: An empirical comparison of small family and non-family businesses. Journal of Management Studies, 45(1), 51 78. doi: 10.1111/j.1467-6486.2007.00718.x 
Miralles-Marcelo, J. L., Miralles-Quirós, M. d. M., \& Lisboa, I. (2014). The impact of family control on firm performance: Evidence from Portugal and Spain. Journal of Family Business Strategy, 5(2), 156-168. doi: 10.1016/j.jfbs.2014.03.002

Mitter, C., Duller, C., Feldbauer-Durstmüller, B., \& Kraus, S. (2014). Internationalization of family firms: the effect of ownership and governance. Review of Managerial Science, 8(1), 1-28. doi: 10.1007/s11846-012-0093-X

Nadkarni, S., \& Herrmann, P. (2010). CEO personality, strategic flexibility, and firm performance: The case of the Indian business process outsourcing industry. Academy of Management Journal, 53(5), 1050-1073. doi: 10.5465/AMJ.2010.54533196

Nielsen, B. B., \& Nielsen, S. (2011). The role of top management team international orientation in international strategic decision-making: The choice of foreign entry mode. Journal of World Business, 46(2), 185-193. doi: 10.1016/j.jwb.2010.05.003

Nitzl, C., Roldan, J. L., \& Cepeda, G. (2016). Mediation analysis in partial least squares path modeling: Helping researchers discuss more sophisticated models. Industrial Management \& Data Systems, 116(9), 1849-1864. doi: 10.1108/imds-07-2015-0302

Ocampo Figueroa, L. E., Alarcón Osuna, M. A., \& Fong Reynoso, C. (2014). Determinants of the internationalization of the firm: The accelerated model vs the sequential model. The International Journal of Business and Finance Research, 8(5), 81-93.

Peiris, I. K., Akoorie, M. E. M., \& Sinha, P. (2012). International entrepreneurship: A critical analysis of studies in the past two decades and future directions for research. Journal of International Entrepreneurship, 10(4), 279-324. doi: 10.1007/s10843-012-0096-3

Pino, R. M. (2008). La relación entre el sector industrial y el tamaño de empresa con las prácticas de calidad total y el desempeño organizacional. (Tesis doctoral), CENTRUM Católica del Perú. Pontificia Universidad Católica del Perú, Lima, Perú. Recuperado de http://tesis.pucp.edu.pe/repositorio/handle/123456789/782

Piva, E., Rossi-Lamastra, C., \& De Massis, A. (2013). Family firms and internationalization: An exploratory study on high-tech entrepreneurial ventures. Journal of International Entrepreneurship, 11(2), 108-129. doi: 10.1007/s10843-012-0100-y

Pukall, T. J., \& Calabrò, A. (2014). The internationalization of family firms: A critical review and integrative model. Family Business Review, 27(2), 103-125. doi: 10.1177/0894486513491423

Reinartz, W., Krafft, M., \& Hoyer, W. D. (2004). The customer relationship management process: Its measurement and impact on performance. Journal of Marketing Research, 41(3), 293-305. doi: 10.1509/jmkr.41.3.293.35991

Salomón, J. O. (2010). Capacidades gerenciales en las empresas familiares peruanas: Un estudio descriptivo. (Tesis doctoral), CENTRUM Católica del Perú. Pontificia Universidad Católica del Perú, Lima, Perú. Recuperado de http://tesis.pucp.edu.pe/repositorio/handle/123456789/1605

Sarstedt, M., Ringle, C. M., \& Hair, J. F. (2014). PLS-SEM: Looking Back and Moving Forward. Long Range Planning, 47(3), 132-137. doi: 10.1016/j.lrp.2014.02.008 
Sarstedt, M., Ringle, C. M., Henseler, J., \& Hair, J. F. (2014). On the Emancipation of PLS-SEM: A Commentary on Rigdon (2012). Long Range Planning, 47(3), 154-160. doi: 10.1016/j.lrp.2014.02.007

Sarstedt, M., Ringle, C. M., Smith, D., Reams, R., \& Hair, J. F. (2014). Partial least squares structural equation modeling (PLS-SEM): A useful tool for family business researchers. Journal of Family Business Strategy, 5(1), 105-115. doi: 10.1016/j.jfbs.2014.01.002

Sciascia, S., Mazzola, P., Astrachan, J. H., \& Pieper, T. M. (2012). The role of family ownership in international entrepreneurship: exploring nonlinear effects. Small Business Economics, 38(1), 1531. doi: 10.1007/s11187-010-9264-9

Segaro, E. (2012). Internationalization of family SMEs: the impact of ownership, governance, and top management team. Journal of Management \& Governance, 16(1), 147-169. doi: 10.1007/s10997010-9145-2

Segaro, E., Larimo, J., \& Jones, M. (2014). Internationalisation of family small and medium sized enterprises: The role of stewardship orientation, family commitment culture and top management team. International Business Review, 23(2), 381-395. doi: 10.1016/j.ibusrev.2013.06.004

Smith, D., Hair, J. F., \& Ferguson, K. (2014). An investigation of the effect of family influence on Commitment-Trust in retailer-vendor strategic partnerships. Journal of Family Business Strategy, 5(3), 252-263. doi: 10.1016/j.jfbs.2013.11.005

Suess, J. (2014). Family governance - Literature review and the development of a conceptual model. Journal of Family Business Strategy, 5(2), 138-155. doi: 10.1016/j.jfbs.2014.02.001

Sullivan, D. (1994). Measuring the degree of internationalization of a firm. Journal of International Business Studies, 25(2), 325-342. doi: 10.1057/palgrave.jibs.8490203

Swoboda, B., \& Olejnik, E. (2013). A taxonomy of small- and medium-sized international family firms. Journal of International Entrepreneurship, 11(2), 130-157. doi: 10.1007/s10843-012-0101-x

Yeoh, P.-L. (2014). Internationalization and performance outcomes of entrepreneurial family SMEs: The role of outside CEOs, technology sourcing, and innovation. Thunderbird International Business Review, 56(1), 77-96. doi: 10.1002/tie.21597

Zahra, S. A., Hayton, J. C., Neubaum, D. O., Dibrell, C., \& Craig, J. (2008). Culture of family commitment and strategic flexibility: The moderating effect of stewardship. Entrepreneurship Theory and Practice, 32(6), 1035-1054. doi: 10.1111/j.1540-6520.2008.00271.x

Zahra, S. A., Neubaum, D. O., \& Naldi, L. (2007). The effects of ownership and governance on SMEs' international knowledge-based resources. Small Business Economics, 29(3), 309-327. doi: 10.1007/s11187-006-9025-y 


\section{Apéndice A: Encuesta Aplicada y Resultados Obtenidos}

Tabla A1. Preguntas y Resultado Obtenido para la Variable Orientación a la Administración En qué medida está usted de acuerdo con las siguientes ideas o afirmaciones.

En qué medida está usted de acuerdo con las siguientes ideas o afirmaciones. 1: No, en absoluto; 5: Totalmente, en gran medida

\begin{tabular}{|c|c|c|c|c|c|c|c|c|c|c|c|c|c|}
\hline \multirow[t]{2}{*}{ Pregunta/Variable } & & \multicolumn{2}{|c|}{1} & \multicolumn{2}{|c|}{2} & \multicolumn{2}{|c|}{3} & \multicolumn{2}{|c|}{4} & \multicolumn{2}{|c|}{5} & \multirow{2}{*}{$\begin{array}{l}\text { Total } \\
\text { casos } \\
\text { válidos }\end{array}$} & \multirow[t]{2}{*}{ Media } \\
\hline & & Frec $^{\mathrm{a}}$ & Porc $^{b}$ & Frec & Porc & Frec & Porc & Frec & Porc & Frec & Porc & & \\
\hline Orientación de lago plazo (LTO) & & & & & & & & & & & & & \\
\hline $\begin{array}{l}\text { (a) Procuramos construir relaciones de largo plazo con } \\
\text { nuestros más importantes proveedores }\end{array}$ & LTO1 & & & & & & & 9 & 7.7 & 108 & 92.3 & 117 & 4.92 \\
\hline $\begin{array}{l}\text { (b) Procuramos construir relaciones de largo plazo con } \\
\text { nuestros más importantes clientes }\end{array}$ & LTO2 & & & & & & & 10 & 8.5 & 107 & 91.5 & 117 & 4.91 \\
\hline $\begin{array}{l}\text { (c) Procuramos construir relaciones de largo plazo con } \\
\text { nuestros principales socios } \\
\text { Orientación a los empleados (EMO) }\end{array}$ & LTO3 & & & & & & & 8 & 6.8 & 109 & 93.2 & 117 & 4.93 \\
\hline $\begin{array}{l}\text { (d) Hasta qué punto la empresa permite que sus } \\
\text { empleados alcancen todo su potencial }\end{array}$ & EMO1 & & & 4 & 3.4 & 17 & 14.5 & 35 & 29.9 & 61 & 52.1 & 117 & 4.31 \\
\hline $\begin{array}{l}\text { (e) Hasta qué punto la empresa fomenta un espacio de } \\
\text { trabajo profesional }\end{array}$ & EMO2 & & & 3 & 2.6 & 12 & 10.3 & 45 & 38.5 & 57 & 48.7 & 117 & 4.33 \\
\hline $\begin{array}{l}\text { (f) Hasta qué punto la empresa inspira lealtad y } \\
\text { compromiso }\end{array}$ & EMO3 & & & & & 4 & 3.4 & 15 & 12.8 & 98 & 83.8 & 117 & 4.80 \\
\hline $\begin{array}{l}\text { (g) Hasta qué punto utilizan a los empleados en la } \\
\text { creación de redes de negocio en nombre y en } \\
\text { representación de la empresa }\end{array}$ & EMO4 & 23 & 19.7 & 7 & 6.0 & 31 & 26.5 & 17 & 14.5 & 39 & 33.3 & 117 & 3.36 \\
\hline Orientación a los clientes (CMO) & & & & & & & & & & & & & \\
\hline $\begin{array}{l}\text { (h) Tenemos un sistema formal para determinar cuáles de } \\
\text { nuestros clientes actuales son más valiosos }\end{array}$ & $\mathrm{CMO1}$ & & & & & 13 & 11.1 & 32 & 27.4 & 72 & 61.5 & 117 & 4.50 \\
\hline $\begin{array}{l}\text { (i) Continuamente damos seguimiento de la información } \\
\text { del cliente para evaluar su valor }\end{array}$ & $\mathrm{CMO} 2$ & & & & & 6 & 5.1 & 16 & 13.7 & 95 & 81.2 & 117 & 4.76 \\
\hline $\begin{array}{l}\text { (j) Activamente tratamos de determinar los costos de } \\
\text { retención de cliente }\end{array}$ & $\mathrm{CMO} 3$ & & & 6 & 5.1 & 4 & 3.4 & 14 & 12.0 & 93 & 79.5 & 117 & 4.66 \\
\hline $\begin{array}{l}\text { (k) Hacemos seguimiento del estado de la relación } \\
\text { durante todo el ciclo de vida del cliente (madurez de la } \\
\text { relación) }\end{array}$ & $\mathrm{CMO4}$ & & & 3 & 2.6 & 9 & 7.7 & 22 & 18.8 & 83 & 70.9 & 117 & 4.58 \\
\hline
\end{tabular}

Nota. a. Frecuencia, b. Porcentaje 
Tabla A2. Preguntas y Resultado Obtenido para la Variable Flexibilidad Estratégica Total

Cuán flexible es la planeación estratégica de su empresa cuándo ésta debe dar respuesta a las siguientes circunstancias. Valore desde 1: Nada flexible; 5: Muy flexible

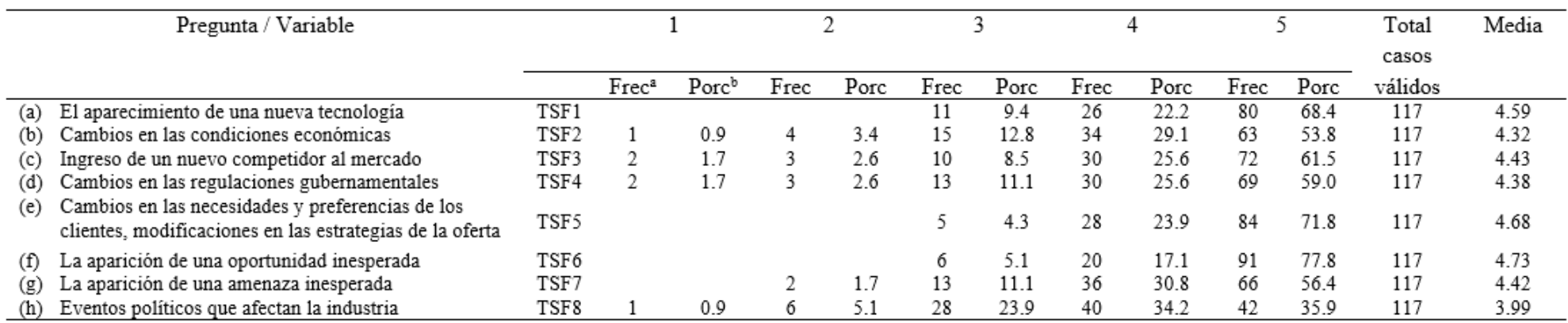

olíticos que afectan la industria TSF8

Nota. ${ }^{\text {a. }}$ Frecuencia, ${ }^{\text {b. }}$ Porcentaje

Tabla A3. Preguntas y Resultado Obtenido para la Variable Heterogeneidad del Equipo Gerencial

\begin{tabular}{lcc}
\hline \multicolumn{1}{c}{ Área de experiencia } & Frec $^{\mathbf{a}}$ & Porc $^{\mathrm{b}}$ \\
\hline (a) Investigación y desarrollo & 26 & 22.2 \\
(b) Producción y operaciones & 47 & 40.2 \\
(c) Marketing y ventas & 32 & 27.4 \\
(d) Finanzas, contabilidad, legal y administrativo & 12 & 10.3 \\
\hline
\end{tabular}

Nota. ${ }^{2}$. Frecuencia, ${ }^{\text {b. Porcentaje }}$

Tabla A4. Resultado General de la Variable Grado de Internacionalización

\begin{tabular}{llccc}
\hline & & & \multicolumn{2}{c}{ Vector } \\
& & & Florícola & Agro industrial \\
\hline (a) & Porcentaje de empleados que dedican más del & EMPIA 50 & Media del $19.9 \%$ & Media del $17.1 \%$ \\
& $50 \%$ a actividades de exportación & FSTSR & Del $70 \%$ al $100 \%$ & Del $10 \%$ al $100 \%$ \\
(b) & Porcentaje de ventas en mercados externos & GESCPR & Del $50 \%$ al $100 \%$ & Del $25 \%$ al $100 \%$ \\
(c) & Ámbito geográfico. Ventas fuera de la CAN & &
\end{tabular}

